\title{
Comparison of Three Methods of Regional Anesthesia of Peripheral Nerves and Plexuses
}

\author{
Valery Piacherski, Aliaksei Marochkov, Andrei Brukhnou, Zakhar Kokhan \\ Department of Anaesthesiology and Intensive Care, Mogilev Regional Hospital, Mogilev, Republic of Belarus. \\ Email: pechersky.v@yandex.ru
}

Received September 19 $9^{\text {th }}, 2012$; revised October $20^{\text {th }}, 2012$; accepted October $29^{\text {th }}, 2012$

\begin{abstract}
Aim: There were acquitted 1105 nerve blocks on 762 patients by means of three methods of peripheral nerves and plexuses identification to compare the safety and efficiency of the methods of regional anesthesia. Methods: Depending on the technique of carrying out the peripheral nerve blocks, patients were divided into 3 groups. 1st group: the identification of the correct placement of the injection needle was done by eliciting paresthesia (572 blocks were performed on 395 patients); 2nd group: an electrical nerve stimulator was used to locate the nerve (164 blocks on 110 patients); 3rd group: the location of the nerve was identified using ultrasonic visual guidance (369 blocks on 257 patients). Results and Conclusion: In 1st group $8(1.4 \%)$ accidental intravascular injections of local anesthetic, 1 case of Horner syndrome $(0.17 \%), 1$ case of phrenical nerve were registered. In 17 cases there were performed other methods of anesthesia by reason of inefficiency of the block. In 2 nd group 1 case $(0.61 \%)$ of intravascular injection was noticed. The block was ineffective in single case. There was no complication received in the 3rd group. All the blocks were effective.
\end{abstract}

Keywords: Regional Anesthesia; Ultrasound-Guided; Efficacy; Complications

\section{Introduction}

Regional anesthesia of peripheral nerve trunks and plexuses is widely used to provide pain relief during operations on limbs.

Undoubtedly, this method of analgesia is considered to be the safest with regard to the development of complications typical for endotracheal narcosis, spinal and epidural anesthesia [1]. Regional blockades of peripheral nerves and plexuses have been widely practised by our anesthesiologists since 1975. During a period of over 30 years we have performed more than 10,000 peripheral nerve and plexus blocks.

Recently, the ultrasonic visual guidance technique has been considered as the global "gold" standard in the regional anesthesia of peripheral trunks and plexuses [2] The ultrasonic visual guidance technique allows you to monitor the spread of local anesthetic to perform a variety of peripheral nerve blocks [3]. The use of ultrasonic visual control during peripheral nerve blocks is considered at the present time to be the best of known technologies for carrying out regional anesthesia, which allows an efficacy of the analgesia of up to $93.1 \%-97.7 \%$ to be achieved at infraclavicular brachial plexus block [4].

The purpose of the present report is to make a comparative evaluation of the outcomes of the regional anesthesia of peripheral nerves and plexuses using a prelimi- nary nerve localization technique with the aid of an electrical nerve stimulator or an ultrasonic visual guidance procedure.

\section{Materials and Methods}

In order to achieve the target, a prospective randomized study of 1105 nerve blocks in 762 patients of both sexes from 1 January 2007 until 1 July 2010 was carried out. All patients underwent operative intervention for lower or upper extremities. Their ages ranged from 18 to 65 years old.

Inclusion criteria. All patients had an operation on the occasion of post-traumatic damage and dysfunction of lower extremities, removal of the metal constructions after bones osteosynthesis in lower extremities, written informed consent after detailed information about the nature, risk, and scope of this clinical study and the desirable and possible adverse effects of the study drug or complications associated with the regional anaesthetic technique. The criterion of exclusion from the research was anomalous sciatic nerve anatomy. Such clinical cases are described separately in an appropriate section of the article. Coagulopathies, lack of cooperation, or other objections in the opinion of the investigators to participate in this study.

The patients were operated on for: 1) post-traumatic 
injuries and functional disorders of lower and upper extremities; 2) vascular pathology of lower and upper extremities (embolectomy, blood vessel damage; 3) amputations of lower and upper limbs in patients with various diseases of vascular, traumatic or purulent inflammatory origin; 4) removal of metal structures from limbs.

Sciatic nerve blocks were carried out by means of posterior, subgluteus, anterior-medial and popliteal approaches. Femoral nerve blocks were performed from the point just below the inguinal ligament with the patient supine. Lumbar plexus blocks were performed by an inguinal approach ("three-in-one" blocking the femoral, obturator and external dermal nerves) with the patient supine, using an injection point distal to the inguinal ligament (as for the femoral nerve block. Brachial plexus blocks were performed by interscalene, supraclavicular, subclavicular or axillary approaches depending on the level and complexity of the operation. Wrist and foot nerve blocks were carried out by infiltrating tissues with a local anesthetic at wrist/ankle level or by injecting a local anesthetic using ultrasonic visual guidance [5].

Patients were divided into 3 groups depending on the method used for location of the nerve and the nerve block technique used.

The 1st group consisted of 395 patients who received 572 blocks. In this group the location of the nerve was determined by the anesthesiologist using anatomical signals until the achievement of paresthesia. The determination of the correct placement of the injection needle was based on the patients' personal sensations: the onset of paresthesia [5].

The identification of the correct placement of the injection needle in relation to the nerve with the aid of paresthesia elicitation was carried out in our hospital up till 2007.

It was connected with the absence of an electric nerve stimulator registration of peripheral nerves in the territory of the Republic of Belarus.

Since 2007 after the acquisition of an electric nerve stimulator, the peripheral nerve blocks consistent with the elicitation of paresthesia have not been practiced by our anaesthesiologists.

The 2 nd group consisted of 110 patients who received 164 blocks. In this group an electrical peripheral nerve stimulator was used to locate the correct placement of the injection needle.

The 3rd group consisted of 257 patients who received 369 blocks. In this group, in order to identify the peripheral nerves and plexuses, to determine the correct needle placement and to control the local anesthetic dispersion during peripheral nerve block procedures, electrical stimulation and ultrasonic visual guidance methods were used. Criteria for the correct placement of the needle were ultrasonic signals of the positioning of the nerve trunk and needle and contractions of the appropriate muscle group. Monitoring of the dispersion of the local anesthetic was carried out using ultrasonic guidance.

The quality of motor blocks was evaluated with the help of the following scale: ++|motion is completely absent; + \motion is not fully retained or it is discoordinated; - motion is fully retained.

The assessment of sensation at the skin was carried out with the help of a similar scale: ++|complete sensory block; +lincomplete sensory block, the patient cannot differentiate the type of irritation; - Isensation at the skin is fully retained.

Patients in the different groups did not differ significantly in age, weight or duration of the operation (Table 1). Physical status of the patients was evaluated on a scale of ASA (Table 2).

The first group consisted of 395 patients who received 572 blocks. In this group the verification of the nerve trunks was carried out by the method of paresthesia location whereby, at the moment when the tip of the needle touched the capsule, the patient loudly "informed" the doctors about the onset of paresthesia.

On the eve of the operation and in the morning on the day of the operation, the following premedication was administered: Diphenhydramine, $50 \mathrm{mg}$ peroral. $30 \mathrm{~min}$ utes prior to the block procedure an intramuscular administration of Atropine $0.5-0.8 \mathrm{mg}$ and Diphenhydramine $10 \mathrm{mg}$, was carried out.

In the operating room additional sedation was not administered as the sedation and the remaining manifestations of "deep" pre-medication complicated cooperation with the patient and verification of the nerve trunks and plexuses.

Patients in the 1st group received: 1) sciatic nerve blocks-177; 2) femoral nerve blocks-177; 3) brachial plexus blocks by an interscalene approach - 133 ; 4) brachial plexus blocks by a supraclavicular approach-34; 5) brachial plexus blocks by an axillary approach $-42 ; 6$ ) wrist nerve blocks by the "cuff" type procedure) - 4; 7) ankle nerve blocks by the "cuff" type procedure- 5 .

Tofizopam (Grandaxin), 50 - $100 \mathrm{mg}$ peroral and Diphenhydramine (Dimedrol), $50 \mathrm{mg}$ peroral were administered to patients in the 2nd group on the eve ( $22.00 \mathrm{hrs})$ of the operation and in the morning (7.00) on the day of the operation. $20-30 \mathrm{~min}$ prior to the block procedure Atropine $0.5-0.8 \mathrm{mg}$ and Diphenhydramine, $10 \mathrm{mg}$ were injected intramuscular.

To provide sedation and to enhance the patient's comfort in the operating room pre-medication with Diazepam, $5-10 \mathrm{mg}$ or Butyrophenone (Droperidol), 2.5 - $5 \mathrm{mg}$ or a combination of Diazepam (the same dose of $5-10 \mathrm{mg}$ ) and Fentanyl, $0.05-0.1 \mathrm{mg}$ was administered.

For verification of the peripheral nerve trunks, an electric nerve stimulator was used and the contractions of 
Table 1. Characteristics of patients in groups $(\mathrm{M} \pm)$.

\begin{tabular}{cccc}
\hline Group & 1 & 2 & 3 \\
\hline No of patients & 395 & 110 & 257 \\
No of anesthesia & 572 & 164 & 369 \\
Age (yrs) & $44.0 \pm 4.1$ & $42.1 \pm 3.3$ & $43.4 \pm 14.6$ \\
Weight, kg & $68.3 \pm 5.2$ & $71.3 \pm 5.4$ & $74.2 \pm 10.5$ \\
Operation duration, min. & $46.9 \pm 5.2$ & $39.2 \pm 6.1$ & $44.7 \pm 7.4$ \\
\hline
\end{tabular}

the appropriate muscle group were observed. The stimulation frequency was $1-2 \mathrm{~Hz}$, pulses were generated at a current intensity of $0.1-1.0 \mathrm{~mA}$ and voltage of $1-10 \mathrm{Wt}$ and pulse width $-0.1 \mathrm{~ms}$. Special disposable insulated needles (covering the whole length of the needle except for the $1 \mathrm{~mm}$ tip) were used to locate the nerve.

Another important criterion to establish correct needle placement was current intensity. At the correct needle placement with respect to the nerve, current intensity was $0.2-0.4 \mathrm{~mA}$ for upper extremity operations and $0.3-0.5$ $\mathrm{mA}$ for lower extremity procedures.

Patients in the 2nd group received: 1) 54 sciatic nerve blocks; 2) 54 femoral nerve blocks ("three-in-one"); 3) 44 brachial plexus blocks by an interscalene approach; 4) 5 brachial plexus blocks by a supraclavicular approach; 5) 7 brachial plexus blocks by an axillary approach.

The 3rd group consisted of 257 patients who received 369 peripheral nerve and plexus blocks. In this group the patients were given pre-medication on the eve of the operation $(22.00 \mathrm{hrs}$.) and in the morning $(7.00 \mathrm{hrs}$.) on the day of the procedure: Tofizopam, $50-100 \mathrm{mg}$ peroral and Diphenhydramine, $50 \mathrm{mg}$ peroral. 20 - $30 \mathrm{~min}$. Prior to the block procedure intramuscular injections of Atropine, $0.5-0.8 \mathrm{mg}$ and Diphenhydramine, $10 \mathrm{mg}$ were administered.

To provide sedation in the operating room prior to the block procedure pre-medication with Diazepam, $10 \mathrm{mg}$, Fentanyl $0.1 \mathrm{mg}$ and/or Propofol 40 - $60 \mathrm{mg}$. was administered. All blocks in the patients of the 3rd group were performed with a $1 \%$ solution of Lidocaine and Adrenaline $(1: 200,000)$, volume $20-40 \mathrm{~mL}$. For the verification of peripheral nerve trunks, an ultrasonic visual guidance technique combined with electric nerve stimulation was used. After verification of the signals of peripheral nerve trunks using the ultrasonic visual guidance technique, the nerve stimulator injection needle was advanced towards the nerve until it resulted in the contraction of the muscles in the appropriate group. The dispersion of the local anesthetic around the nerve could be observed on the monitor screen. In order to achieve a visual signal of the nerve trunks, an ultrasonic Linear Probe at a frequency of 3.5 - 7.5 MHz was used [6].

Patients in the 3rd group were given the following blocks: 1) sciatic nerve block by a subgluteal approach $58 ; 2)$ sciatic nerve block by a popliteal approach $-19 ; 3$ ) sciatic nerve block by an anterior-medial approach-35; 4) femoral nerve block-112; 5) brachial plexus block by an interscalene approach-96;6) brachial plexus block by a supraclavicular approach-14; 7) brachial plexus block by a subclavicular approach $-1 ; 8$ ) brachial plexus block by an axillary approach-31;9) foot nerve block at ankle level (cuff type)-3.

The peripheral vein was catheterized in all patients on the operating table. Blocks in the patients of all three groups were performed with a $1 \%$ solution of Lidocaine and Adrenaline (1:200,000), volume $20-40 \mathrm{~mL}$.

The efficacy and safety of the blocks were evaluated on the basis of the following criteria:

- Sedation estimation (according to Ramsay et al., 1974)

[7];

- Number of complications;

- Number of ineffective blocks.

During operations all patients were monitored by ECG, heart rate, respiratory rate, non-invasive arterial blood pressure, pulseoximetry and thermometry measurements.

\section{Ethics}

Ethical approval for this study was provided by the Ethical Committee of the Mogilev Regional Hospital, Belarus (President Dr. Alexandr R. Stolin), Protocol No. 3/C on 2 August 2010.

\section{Results}

Processing of the statistical data was carried out on a PC using the "MS EXCEL" package for statistical analysis.

213 patients from the 1st group did not receive sedation treatment before the block procedure. The sedation was administered to 182 patients and it was evaluated according to Ramsay et al. [7] as sedation stage II (Table 3).

This was associated with the need to involve the patient during the block procedure, whereby the patient should inform the anesthesiologist about his personal perception during the localization of the nerve and the elicitation of paresthesia. 
Table 2. Physical status assessment according to ASA.

\begin{tabular}{cccc}
\hline ASA & 1st group $(\mathrm{n}=395)$ & 2nd group $(\mathrm{n}=110)$ & 3rd group $(\mathrm{n}=257)$ \\
\hline 1 class & $76(19.24 \%)$ & $24(21.82 \%)$ & $102(39.6 \%)$ \\
2 class & $201(50.90 \%)$ & $59(53.64 \%)$ & $143(55.64 \%)$ \\
3 class & $77(19.49 \%)$ & $18(16.36 \%)$ & $12(4.76 \%)$ \\
4 class & $41(10.37 \%)$ & $9(8.18 \%)$ & $0(0 \%)$ \\
\hline
\end{tabular}

Table 3. Evaluation of sedation stage according to Ramsay scale.

\begin{tabular}{|c|c|c|c|}
\hline Sedation stage & 1st group $(\mathrm{n}=395)$ & 2 nd group $(n=110)$ & 3 rd group $(n=257)$ \\
\hline I & $213(53.9 \%)$ & 0 & 0 \\
\hline II & $182(46.1 \%)$ & $9(8.2 \%)$ & 0 \\
\hline III & 0 & $83(75.4 \%)$ & $41(15.9 \%)$ \\
\hline IV & 0 & $18(16.4 \%)$ & $169(65.7 \%)$ \\
\hline V & 0 & & $45(18.4 \%)$ \\
\hline
\end{tabular}

Note: Sedation stage (according to Ramsay, 1974) [4]: 1) Patient is awake, restless, excitable and/or impatient; 2) Patient is awake, quiet, oriented, cooperative with the physician; 3) Patient is conscious but responds only to commands; 4) Patient is asleep but responds actively to a touch or a loud sound;5) Patient is asleep and responds flaccidly to loud sounds or tactile stimuli; 6) Patient is asleep and does not respond to commands [4].

In the 2nd group there were only $8.18 \%$ of patients with stage I sedation according to Ramsay et al. [4]. The use of an electrical peripheral nerve stimulator for the nerve blocks in the patients of the 2nd group made it possible to achieve deeper sedation: namely, patients with stage III sedation comprised $75.4 \%$ and those with stage IV sedation-16.3\%. There was no need for the anesthesiologist to obtain paresthesia as a source of information on the optimal placement of the needle in relation to the nerve capsule.

However, in the 1st and 2nd groups it was necessary to have the patients with various sedation levels awake at the time of performing the block because during a test injection of a local anesthetic, an intraneural injection could occur, although unlikely. The patients had to be able to answer the anesthesiologist's question "do you have burning pains along the course of the nerve?" at the moment of administration of Lidocaine. The occurrence of such a "burning pain" during the test injection of the anesthetic would indicate an intraneural dispersion. Under such circumstances patients in the 1st and 2nd groups retained the "effect of presence" in the operating room.

Patients in the 3rd group reached stage IV and V sedation according to Ramsay et al. in $67.5 \%$ and $18.4 \%$ respectively of cases before the block was carried out. Stage III sedation was determined in $15.9 \%$ of cases. For the verification of peripheral nerve trunks and correct needle placement there was no necessity for the patient's cooperation. Visual control of the dispersion of the local anesthetic made it possible to avoid intraneural injection without verbal communication with the patient.
The use of "deeper" sedation for patients in the 3rd group created, from our point of view, the best conditions for performing peripheral nerve blocks (Table 3).

The need for the use of ultrasonic visual control during the performance of peripheral nerve blocks is associated with the higher quality of pain relief achieved by use of the new method. We have chosen a clinical criterion as an objective one for the estimation of the efficacy of the ultrasonic visual control technique-which allows the possibility to use other types of anesthesia in case of failure or inadequate quality of the peripheral nerve block.

The number of ineffective blocks (2.97\%) was considerably (4 times) higher in the 1st group than in the 2 nd $(0.64 \%)$. Ineffective blocks in the 2 nd group were possibly related to the displacement of the needle whilst injecting the anesthetic since a motor response with 0.3 $\mathrm{mA}$ current intensity and $2 \mathrm{~Hz}$ frequency was obtained by the anesthesiologist.

Failed blocks were not observed in the 3rd group. This is related to the successful localization of the nerve using an ultrasonic control technique in combination with electrical nerve stimulation. Ultrasonic control made it possible to observe the dispersion of the local anesthetic and needle placement and to monitor the latter if it appeared to be displaced at the moment of introducing the injectate. (Table 4).

The new method for performing peripheral nerve and plexus blocks makes it possible to avoid such complications as local anesthetic intravascular injection, pneumothorax, high spinal anesthesia, stellate ganglion block, 
Table 4. Quality evaluation of regional blocks.

\begin{tabular}{cccc}
\hline Using another type of analgesia & $\begin{array}{c}1 \text { st group } \\
(572 \text { blocks })\end{array}$ & 2nd group (164 blocks) & $\begin{array}{c}\text { 3rd group } \\
(369 \text { blocks })\end{array}$ \\
\hline Inravenous narcosis & $14(2.45 \%)$ & $1(0.61 \%)$ & 0 \\
Endotracheal narcosis & $2(0.35 \%)$ & 0 & 0 \\
Spinal anesthesia & $1(0.17 \%)$ & 0 & 0 \\
Total & $17(2.97 \%)$ & $1(0.61 \%)$ & $0 \%$ \\
\hline
\end{tabular}

Horner's syndrome, phrenic nerve block and others.

Various incidences of the above-mentioned complications (Table 5) were noted in the 1st group. The total number of such complications in the 1st group was 14 (2.45\%).

A single case of intravascular injection of the local anesthetic $(0.61 \%)$ was noted in the 2 nd group.

No complications with regional nerve blocks were observed in the 3rd group (Table 5).

The study carried out by us has shown that the use of ultrasonic visual control of the peripheral nerve trunks, plexuses and needle placement during the performance of peripheral nerve blocks makes it possible to improve the safety of the anesthesia procedure, to avoid complications and the "presence effect" of the patient in the operation room.

\section{Discussion}

But, despite all this, the efficiency and safety of performing blocks was high. There were no additional methods of anesthesia performed in 3rd group. In the 1st group there was no purpose to use more deep sedation for performing the block, because the inhibited reaction and the consciousness disorders would considerably trouble the identification of the injection needle about the nerve. So, patient had to inform the anaesthetist in case of paresthesia. Using the paresthesia scanning method, the optimal sedation level was evaluated according to Ramsay et al. [7] as sedation stage I - II. However, some patients on the sedation stage II had shown the decrease of the answer rapidity to paresthesia, what discommoded an identification of the injection needle location. In the second group, patients had been having the sedation stage III - IV. Patients in the 3rd group reached stage IV and V sedation according to Ramsay et al. The use of "deeper" sedation created the best conditions for performing peripheral nerve blocks. Though, it was necessary to keep contacting the patient in the 2 nd group to detect accidental intraneural injection of anesthetic - patient must inform the anaesthetist about the severe pain at the time of injection. Using ultrasound visualization, the expansion of local anesthetic was controlling, what saved from necessity to contact the patient and allowed to use more adequate sedation. Applying blocks with paresthesia scanning, the complications were minimal. This fact may be determined by high qualification and experience of the anaesthetist. Considering the literature data, the phrenical nerve block is in progress almost in all the cases of performing the brachial plexus block by means of interscalene approach using $30-40 \mathrm{~mL}$ of anesthetic $[1,3]$. Consequently, we appraised as a complication only the one case of phrenical nerve block, when respiratory insufficiency had developed and the patient had been observing at the Anaesthesiology and Reanimation Department. On the morrow of the local anesthetic effect, the symptoms of respiratory insufficiency were eliminated. The patient was transferred to the traumathological department safely. One case of pneumothorax was fixed at the realization of brachial plexus block by supraclavicular approach.

A reliable verification of the location of the nerve trunks, plexuses and injection needle placement with the aid of the ultrasonic visual control technique makes it possible to avoid various complications during regional anesthesia of peripheral nerves and plexuses and to get a notable increase of its efficiency. Perhaps, high efficiency of blocks using ultrasonic visual guidance may be obtained even by anesthetists having a moderate experience, as it was shown on the 3rd group, but this question has to be studied more thoroughly.

213 patients from the 1st group did not receive sedation treatment before the block procedure. The sedation was administered to 182 patients and it was evaluated according to Ramsay et al. [4] as sedation stage II (Table 3).

This was associated with the need to involve the patient during the block procedure, whereby the patient should inform the anesthesiologist about his personal perception during the localization of the nerve and the elicitation of paresthesia.

In the 2 nd group there were only $8.18 \%$ of patients with stage I sedation according to Ramsay et al. [7]. The use of an electrical peripheral nerve stimulator for the nerve blocks in the patients of the 2nd group made it possible to achieve deeper sedation: namely, patients with stage III sedation comprised $75.4 \%$ and those with 
Table 5. Complications.

\begin{tabular}{cccc}
\hline Complication & $\begin{array}{c}\text { 1st group } \\
(572 \text { blocks })\end{array}$ & 2nd group (164 blocks) & $\begin{array}{c}\text { 3rd group } \\
(369 \text { blocks })\end{array}$ \\
\hline Intravascular injection & $8(1.4 \%)$ & $1(0.61 \%)$ & 0 \\
Pneumothorax & $1(0.17 \%)$ & 0 & 0 \\
High spinal anaesthesia & $1(0.17 \%)$ & 0 & 0 \\
Horner's syndrome & $3(0.52 \%))$ & 0 & 0 \\
Phrenic nerve block & $1(0.17 \%)$ & 0 & 0 \\
Total & $14(2.45 \%)$ & $1(0.61 \%)$ & 0 \\
\hline
\end{tabular}

stage IV sedation- $16.3 \%$. There was no need for the anesthesiologist to obtain paresthesia as a source of information on the optimal placement of the needle in relation to the nerve capsule.

However, in the 1st and 2nd groups it was necessary to have the patients with various sedation levels awake at the time of performing the block because during a test injection of a local anesthetic, an intraneural injection could occur, although unlikely. The patients had to be able to answer the anesthesiologist's question "do you have burning pains along the course of the nerve?" at the moment of administration of Lidocaine. The occurrence of such a "burning pain" during the test injection of the anesthetic would indicate an intraneural dispersion. Under such circumstances patients in the 1 st and 2 nd groups retained the "effect of presence" in the operating room.

Patients in the 3rd group reached stage IV and V sedation according to Ramsay et al. in $67.5 \%$ and $18.4 \%$ respectively of cases before the block was carried out. Stage III sedation was determined in $15.9 \%$ of cases. For the verification of peripheral nerve trunks and correct needle placement there was no necessity for the patient's cooperation. Visual control of the dispersion of the local anesthetic made it possible to avoid intraneural injection without verbal communication with the patient.

The use of "deeper" sedation for patients in the 3rd group created, from our point of view, the best conditions for performing peripheral nerve blocks (Table 3).

The need for the use of ultrasonic visual control during the performance of peripheral nerve blocks is associated with the higher quality of pain relief achieved by use of the new method. We have chosen a clinical criterion as an objective one for the estimation of the efficacy of the ultrasonic visual control technique-which allows the possibility to use other types of anesthesia in case of failure or inadequate quality of the peripheral nerve block.

The number of ineffective blocks $(2.97 \%)$ was considerably (4 times) higher in the 1 st group than in the 2 nd $(0.64 \%)$. Ineffective blocks in the 2 nd group were possibly related to the displacement of the needle whilst in- jecting the anesthetic since a motor response with 0.3 $\mathrm{mA}$ current intensity and $2 \mathrm{~Hz}$ frequency was obtained by the anesthesiologist.

Failed blocks were not observed in the 3rd group. This is related to the successful localization of the nerve using an ultrasonic control technique in combination with electrical nerve stimulation. Ultrasonic control made it possible to observe the dispersion of the local anesthetic and needle placement and to monitor the latter if it appeared to be displaced at the moment of introducing the injectate (Table 4).

A reliable verification of the location of the nerve trunks, plexuses and injection needle placement with the aid of the ultrasonic visual control technique makes it possible to avoid various complications during regional anesthesia of peripheral nerves and plexuses.

Stage IV and V sedation levels achieved in accordance with the Ramsay scale eliminated the "presence effect" of the patient in the operating room and thus saved the patient from unpleasant experiences during the block procedure.

The reviews of the scientific literature indicate that the effectiveness of the peripheral blocks using ultrasound guidance is in the range $80 \%$ [8]. It is these performance measures do not allow us to call a method of "gold standard". In our view, these figures are very strange. Anesthesiologist can monitor the whole process of implementation of the blockade, adjust the position of a needle, to influence the nature of the spread of local anesthetic. If the anesthesiologist monitors the wrong distribution of anesthetic around the nerve, he can change it. Incorrect injection of local anesthetic is one of the main reasons for the ineffectiveness of regional anesthesia. Ultrasound guidance should rectify this regional anesthesia. It is unclear why the visualization of the whole process of the blockade and the ability to change the place of administration of local anesthetic blockade of peripheral efficiency remains low (80\%). However, patient satisfaction is also an important factor in the clinical acceptability of a technique which will depend on whether it provides a pain-free and predictably successful outcome [8]. Our 
results showed that the use of ultrasound for regional blocks has the potential to raise the standard and to drive forward success rates and safety. In our view, the main way to improve the efficiency of peripheral blocks under ultrasound guidance is to continuously monitor the spread of the local anesthetic and timely correction when incorrect.

\section{Acknowledgements}

Hadun Lyibov, Grafova Svetlana.

\section{REFERENCES}

[1] M. Malroj, "Regional Anaesthesia: Illustrated Practice Book," 3rd Edition, Wolters Kluwer/LippincottWilliams \& Wilkins, Philadelphia, 2002, p. 333.

[2] G.-J. van Geffen, "The Value of Ultrasonography for Performing Peripheral Nerve Blocks," Theory, Practice and Clinical Experience in Adults and Children, Optima Grafische Communicatie, Rotterdam, 2008, pp. 234-279.

[3] M. K. Son, R. K. Chung, Y. J. Kim, D. Y. Kim, H. S. Lee and J. I. Han, "The Effects of Local Anesthetic Distribu- tion on Symptoms Using Ultrasound Image after Stellate Ganglion Ock," Korean Journal of Anesthesiology, Vol. 57, No. 5, 2009, pp. 579-583. doi:10.4097/kjae.2009.57.5.579

[4] H. T. De Quang, R. Gianluca, M. Loreto, Z. Cedrick and J. F. Roderick, "A Prospective, Randomized Comparison between Single- and Double-Injection Ultrasound-Guided Infraclavicular Brachial Plexus Block," Regional Anesthesia \& Pain Medicine, Vol. 35, 2010, pp. 16-21.

[5] J. H. Ramphell, M. N. Joseph and M. V. Cristopher, "Regional Anaesthesia: The Requisites in Anaesthesiology," Elsevier Mosby, Maryland Heights, 2004, p. 218.

[6] J. E. Chelly, "Peripherial Nerve Blocks: A Color Atlas," 3rd Edition, Wolters Kluwer/LippincottWilliams \& Wilkins, Philadelphia, 2009, pp. 1-474.

[7] G. Duke, "AnaesthesiaSecres," 4th Edition, Elsevier Mosby, Maryland Heights, 2011, p. 574.

[8] P. Marhofer, W. Harrop-Griffiths, et al., "Fifteen Years of Ultrasound Guidance in Regional Anaesthesia: Part 1," British Journal of Anaesthesia, Vol. 104, No. 5, 2010, pp. 538-546. doi:10.1093/bja/aeq069 